Geometry $\&$ Topology

Volume 9 (2005) 299-313

Published: 28 January 2005

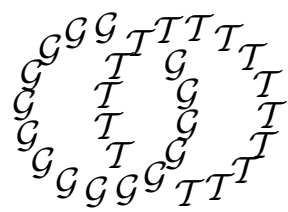

\title{
Homologie de contact des variétés toroïdales
}

\author{
FrÉDÉRIC BOURGEOIS \\ VinCEnt COLIN \\ Université Libre de Bruxelles, Département de Mathématiques CP 218 \\ Boulevard du Triomphe, 1050 Bruxelles, Belgium \\ and \\ Université de Nantes, Laboratoire de Mathématiques Jean Leray \\ 2 rue de la Houssinière, BP 92208, 44322 Nantes Cedex 3, France
}

Email: fbourgeo@ulb.ac.be and Vincent.Colin@math.univ-nantes.fr

\begin{abstract}
We show that contact homology distinguishes infinitely many tight contact structures on any orientable, toroidal, irreducible 3-manifold. As a consequence of the contact homology computations, on a very large class of toroidal manifolds, all known examples of universally tight contact structures with nonvanishing torsion satisfy the Weinstein conjecture.

\section{Résumé}

On montre que l'homologie de contact distingue une infinité de structures de contact tendues sur toute variété toroïdale irréductible et orientable de dimension trois. En conséquence des calculs d'homologie de contact, sur une très large classe de variétés toroïdales, tous les exemples de structures de contact universellement tendues de torsion non nulle connus vérifient la conjecture de Weinstein.
\end{abstract}

\section{AMS Classification numbers Primary: 53D35}

Secondary: $53 \mathrm{C} 15$

Keywords: Contact structures, Reeb fields, contact homology, toroidal manifolds, Weinstein conjecture

Proposed: Yasha Eliashberg

Seconded: Leonid Polterovich, David Gabai
Received: 25 November 2004

Accepted: 24 January 2005 


\section{Introduction}

Dans cet article, on prouve que l'homologie de contact distingue une infinité de structures de contact tendues sur toute variété toroïdale, irréductible et orientable de dimension trois.

Théorème 1.1 (Voir aussi [6, 7], 14]) Toute variété toroïdale, irréductible, orientable, close de dimension trois porte une infinité de structures de contact hypertendues deux à deux non isotopes et distinguées par leur homologie de contact.

Cette approche démontre que l'homologie de contact [11] détecte la torsion [12, 6] dans son cadre le plus général. La famille de structures de contact étudiée est la même que dans 6, 7] et [14. Ici, on montre que tous ses membres sont deux à deux non isotopes, alors qu'avec les arguments de [6, 7, 14, on obtient seulement l'existence d'une sous-famille infinie non explicite ayant cette propriété. En revanche, on n'obtient pas en général que les structures considérées sont deux à deux non isomorphes.

Dans le cas des fibrés en tores sur le cercle, l'homologie de contact est toujours de rang infini. On définit alors un invariant numérique en enrichissant l'homologie de contact au moyen de la fonctionnelle d'action pour distinguer une infinité de structures de contact sur ces variétés.

Les exemples étudiés sont les seules structures de contact universellement tendues connues - et conjecturalement les seules - sur les variétés toroïdales irréductibles. On montre ici (théorème 4.1) que, parmi celles-ci, sur "presque toutes les variétés", toutes celles de torsion non nulle ont une homologie de contact non nulle. En particulier, elles vérifient la conjecture de Weinstein: leurs champs de Reeb possèdent tous une orbite périodique. Par ailleurs, dans ce cas, les structures non universellement tendues sont virtuellement vrillées, et vérifient également, d'après Hofer [13, la conjecture de Weinstein. D'après [8], les structures de contact tendues de torsion nulle sur une variété donnée sont en nombre fini; d'après ce qui précède, ce sont potentiellement les seules pour lesquelles la conjecture de Weinstein reste ouverte. Une preuve de la conjecture de Weinstein pour les structures de contact portées par un livre ouvert planaire a récemment été annoncée par Abbas, Cieliebak et Hofer 11. Il est vraisemblable que les structures de contact tendues de torsion non nulle soient toutes de genre supérieur à 1, auquel cas nos exemples seraient disjoints de ceux de 1].

Une structure de contact $\xi$ est hypertendue si elle possède un champ de Reeb $R$ sans orbite périodique contractible. Dans le cas où la variété $V$ est toroïdale, 
toutes les orbites de $R$ sont d'ordre infini dans le groupe fondamental $\pi_{1}(V)$. En particulier, tout rappel de $\xi$ dans un revêtement fini de $V$ est hypertendu, et donc tendu d'après Hofer [13. Lorsque $V$ est torö̈dale, $\pi_{1}(V)$ est résiduellement fini, et la structure $\xi$ universellement tendue.

Toutes les variétés considérées par la suite sont orientées, et les structures de contact positives pour cette orientation. Les bords sont orientés par la règle de la "normale sortante en premier". Si $A$ est une variété orientée, $-A$ désigne la variété $A$ munie de l'orientation opposée.

Remerciements VC remercie le CNRS pour son accueil en délégation au cours de l'année 2003-2004.

\section{Construction d'une famille infinie}

Dans cette section, on décrit la construction des structures de contact non isotopes dans le théorème 1.1 Pour plus de simplicité, on se restreint au cas où la torsion apparaît dans une seule classe d'isotopie de tore. L'énoncé général est rejeté la section 4

On part d'une variété $V$ irréductible, toroïdale, close et orientée de dimension trois. Soit $T \subset V$ un tore incompressible et $T^{2} \times[a, b]$ un voisinage tubulaire de $T \simeq T^{2} \times\{(a+b) / 2\}$. On note $V^{\prime}$ la variété $\left.V^{\prime}=V \backslash T^{2} \times\right] a, b[$. On se donne une surface essentielle minimale coorientée $S$ dans $V^{\prime}$ (i.e. incompressible, bordincompressible, sans disque parallèle au bord, sans composante close, et qui minimise la norme de Thurston dans $\left.H_{2}\left(V^{\prime}, \partial V^{\prime}, \mathbb{Z}\right)\right)$, qui rencontre les deux composantes $T^{2} \times\{a, b\}$ de $\partial V^{\prime}$.

On a besoin de mesurer des pentes dans $T^{2} \times[a, b+2 k \pi], k \in \mathbb{Z}$. Pour cela, on choisit une base $([l],[m])$ de $H_{1}\left(T^{2}, \mathbb{Z}\right)$, et on considère la projection

$$
p: T^{2} \times[a, b+2 k \pi] \rightarrow T^{2}=(\mathbb{R} / \mathbb{Z})^{2}:((x, y), t) \rightarrow(x, y)
$$

de fibre $[a, b+2 k \pi]$. À toute courbe non contractible $\gamma$ dans $T^{2} \times[a, b+2 k \pi]$, on associe sa pente égale à $(p, q) / \sqrt{p^{2}+q^{2}} \in S^{1} \subset \mathbb{R}^{2}$, où $p$ et $q$ sont définis par l'identité

$$
[p(\gamma)]=p[l]+q[m] \in H_{1}(V, \mathbb{Z}) .
$$

Comme $\gamma$ est orientée, elle est aussi coorientée. Son cône positif $C(\gamma)$ est le demi-cercle fermé $\left(\subset S^{1}\right)$, délimité par les pentes de $\gamma$ et $-\gamma$, et contenant les pentes des courbes $\delta$ de $T^{2}$ qui ont une intersection homologique $p(\gamma) \cdot \delta$ positive.

Une fois ces notations en place, on se repose sur la construction suivante [9]: 
Théorème 2.1 Pour tout $\epsilon>0$, il existe une forme de contact hypertendue $\alpha_{\epsilon}$ sur $V$ et deux réels $c, c^{\prime}>0$ avec les propriétés suivantes:

- le champ de Reeb $R_{\epsilon}$ de $\alpha_{\epsilon}$ est positivement transversal à $S$, tangent à $T^{2} \times\{a, b\}$;

- $R_{\epsilon}$ vaut $\cos t \partial_{x}-\sin t \partial_{y}$ sur $T^{2} \times\left[a+c, b-c^{\prime}\right]\left(\alpha_{\epsilon}\right.$ vaut $\left.\cos t d x-\sin t d y\right)$;

- $\operatorname{sur} T^{2} \times[a, a+c]$ et $T^{2} \times\left[b-c^{\prime}, b\right], R_{\epsilon}$ est positivement transversal à un feuilletage en anneau $\mathcal{F}_{a} \times[a, a+c] \cup\left(-\mathcal{F}_{b}\right) \times\left[b-c^{\prime}, b\right]$, où $\mathcal{F}_{a}$ et $\mathcal{F}_{b}$ sont des feuilletages en cercles de $T^{2} \times\{a\}$ et $T^{2} \times\{b\}$ dont les pentes sont $\epsilon$-proches de celles d'une composante de, respectivement, $\partial S \cap T^{2} \times\{a\}$ et $\partial S \cap T^{2} \times\{b\}$.

On peut en particulier trouver une forme de contact hypertendue $\alpha_{\epsilon}$ qui satisfait aux conclusions du théorème 2.1 avec de surcroît $(b-a) /(2 \pi) \leq 1$. Pour tout $k \in \mathbb{N}$, on note alors $\alpha_{\epsilon, k}$ la forme de contact hypertendue obtenue en remplaçant dans $V$ le produit de contact $\left(T^{2} \times\left[a+c, b-c^{\prime}\right], \cos t d x-\sin t d y\right)$ par $\left(T^{2} \times\left[a+c, b-c^{\prime}+2 k \pi\right], \cos t d x-\sin t d y\right)$. Les formes $\alpha_{\epsilon, k}$ sont hypertendues, donc les structures $\xi_{\epsilon, k}=\operatorname{ker} \alpha_{\epsilon, k}$ sont universellement tendues.

On montre que, pour $\epsilon$ assez petit fixé, les structures $\xi_{\epsilon, k}$ sont, dans la plupart des cas, deux à deux non isotopes.

\section{Preuve du théorème 1.1}

La preuve utilise l'homologie de contact, définie dans la section 3.1 et est subdivisée de la manière suivante. Dans la section 3.2 on étudie le cas où la pente de $\partial S \cap T^{2} \times\{a\}$ est différente de celle de $\partial S \cap T^{2} \times\{b\}$, dans la section 3.3 le cas où ces pentes sont identiques, et dans la section 3.4 le cas des fibrés en tores sur le cercle et des variétés obtenues par collage de deux fibrés tordus en intervalles sur la bouteille de Klein.

\subsection{Homologie de contact}

L'homologie de contact est un invariant des structures de contact introduit par Eliashberg, Givental et Hofer [11. Nous utiliserons ici la version la plus simple de cet invariant : l'homologie de contact cylindrique.

Pour la définir, on fixe une classe d'homotopie libre de lacets $h$ de $V$, et on considère le $\mathbb{Z}$-module $C_{*}^{h}$ librement engendré par les orbites périodiques de $R_{\epsilon, k}$ 
dans la classe $h$. Si la forme de contact $\alpha_{\epsilon, k}$ est choisie de manière générique, ces orbites périodiques sont non dégénérées, et on peut associer à chaque orbite $\gamma$ son indice de Conley-Zehnder $\mu(\gamma)$. La graduation de $\gamma$ dans le module $C_{*}^{h}$ est donnée par $\mu(\gamma)-1$.

On munit la structure de contact $\xi_{\epsilon, k}$ d'une structure complexe $J$ compatible avec la forme symplectique $d \alpha_{\epsilon, k}$. On étend ensuite $J$ en une structure presque complexe, toujours notée $J$, sur la symplectisation $\left(\mathbb{R} \times V, d\left(e^{t} \alpha_{\epsilon, k}\right)\right)$ de $\left(V, \xi_{\epsilon, k}\right)$, en posant $J \frac{\partial}{\partial t}=R_{\epsilon, k}$. On considère les espaces de modules des cylindres $J-$ holomorphes dans $\left(\mathbb{R} \times V, d\left(e^{t} \alpha_{\epsilon, k}\right)\right)$, convergeant lorsque $t \rightarrow \pm \infty$ vers des orbites périodiques de $R_{\epsilon, k}$ dans la classe $h$.

Lorsque la classe $h$ ne contient que des orbites simples de $R_{\epsilon, k}$, on peut choisir $J$ génériquement pour que ces espaces de modules soient des variétés lisses [10]. Comme les formes $\alpha_{\epsilon, k}$ sont hypertendues, leurs champs de Reeb $R_{\epsilon, k}$ ne comptent aucune orbite périodique contractible, et donc les cylindres $J$ holomorphes ne peuvent dégénérer en faisant apparaître un plan complexe. Par conséquent, les espaces de modules considérés sont compacts [4]. En particulier, l'homologie de contact cylindrique est bien définie dans cette situation.

On définit une différentielle linéaire $d: C_{*}^{h} \rightarrow C_{*-1}^{h}$ au moyen des cylindres $J$-holomorphes dans la symplectisation $\left(\mathbb{R} \times V, d\left(e^{t} \alpha_{\epsilon, k}\right)\right)$, de sorte que le coefficient de $\gamma_{2}$ dans $d \gamma_{1}$ compte le nombre de cylindres $J$-holomorphes rigides convergeant vers $\gamma_{1}$ lorsque $t \rightarrow+\infty$ et vers $\gamma_{2}$ lorsque $t \rightarrow-\infty$. Les espaces de modules sont orientés [5] de manière cohérente, de manière à pouvoir compter les cylindres $J$-holomorphes rigides avec un signe.

Cette différentielle $d$ satisfait $d^{2}=0$, en vertu d'un théorème de collage [3] très semblable à celui utilisé pour l'homologie de Floer. On a ainsi défini un complexe $\left(C_{*}^{h}, d\right)$. L'homologie $H C_{*}^{h}(V, \xi, \mathbb{Z})$ de ce complexe, appelée homologie de contact cylindrique, est un invariant de la classe de conjugaison de $\xi$ par tout difféomorphisme qui fixe $h$, et en particulier par isotopie.

On montre que pour une classe $h$ bien choisie, les seules orbites périodiques dans $h$ proviennent du produit $T^{2} \times\left[a+c, b-c^{\prime}+2 k \pi\right]$. On en déduit un calcul explicite de $H C_{*}^{h}\left(V, \xi_{\epsilon, k}, \mathbb{Z}\right)$ (corollaire 3.3 ).

Dans les sections 3.2 et 3.3, la variété $V$ n'est pas un fibré en tores sur le cercle ni obtenue par collage de deux fibrés tordus en intervalles sur la bouteille de Klein. L'étude de ces deux cas est rejetée dans la section [3.4 


\subsection{La pente de $\partial S \cap T^{2} \times\{a\}$ est différente de celle de $\partial S \cap T^{2} \times\{b\}$}

Dans ce cas, on peut choisir $\epsilon$ assez petit de sorte que le secteur

$$
H=S^{1} \backslash\left(C\left(\partial S \cap T^{2} \times\{a\}\right) \cup C\left(-\left(\partial S \cap T^{2} \times\{b\}\right)\right) \cup C\left(\mathcal{F}_{a}\right) \cup C\left(-\mathcal{F}_{b}\right)\right)
$$

soit de cardinal infini.

Dans les lemmes qui suivent, on oriente les composantes de bord de tout anneau $A$ dans le même sens de sorte qu'elles soient librement homotopes dans $A$.

Lemme 3.1 On suppose qu'aucune composante de $V^{\prime}$ n'est difféomorphe à $T^{2} \times[0,1]$ ni à un fibré tordu en intervalles sur la bouteille de Klein. Il existe des pentes $p_{a}$ et $p_{b}$ telles que, pour tout anneau $A$ immergé dans $V^{\prime}, \partial A \subset \partial V^{\prime}$, les pentes des deux composantes de $\partial A \subset T^{2} \times[a, b]$ appartiennent toutes deux à $\left\{ \pm p_{a}, \pm p_{b}\right\}$, ou soient identiques.

Démonstration Si $A$ n'est pas essentiel, il est homotope à un anneau inclus dans $\partial V^{\prime}$ et les pentes de ses deux composantes de bord sont identiques.

Sinon, $A$ est homotope à un anneau $A^{\prime}$ inclus dans une composante $V^{\prime \prime}$ de la décomposition de Jaco-Shalen-Johanson de $V^{\prime}$ qui est un fibré de Seifert (d'après le Mapping Theorem de Jaco-Shalen [15). Les fibres de $V^{\prime \prime}$ rencontrent $T^{2} \times\{a\}$ ou $T^{2} \times\{b\}$; on note $p_{a}$ ou $p_{b}$ leur pente dans $T^{2} \times[a, b]$.

Par hypothèses, $V^{\prime \prime}$ n'est pas un fibré tordu en intervalles sur une bouteille de Klein ni le tore épais ni un tore solide. D'après Jaco-Shalen (Lemme II.2.8, [15]), les pentes des composantes de bord de $A$ sont $\pm p_{a}$ où $\pm p_{b}$.

Soit $\gamma_{0}$ une courbe de $T^{2} \times[a, b]$ dont la pente est dans $H \backslash\left\{ \pm p_{a}, \pm p_{b}\right\}$. On note $h$ sa classe d'homotopie libre.

Lemme 3.2 Si aucune composante de $V^{\prime}$ n'est un tore épaissi ou un fibré tordu en intervalles sur la bouteille de Klein, toute orbite de $R_{\epsilon, k}$ dans la classe $h$ est incluse dans

$$
T^{2} \times\left[a+c, b-c^{\prime}+2 k \pi\right]
$$

et est de même pente que $\gamma_{0}$.

Démonstration Soit $\gamma_{1}$ une orbite périodique de $R_{\epsilon, k}$ librement homotope à $\gamma_{0}$. Elle est incluse soit dans $T^{2} \times\left[a+c, b-c^{\prime}+2 k \pi\right]$, soit dans $T^{2} \times([a, a+$ $\left.c] \cup\left[b-c^{\prime}+2 k \pi, b+2 k \pi\right]\right)$, soit dans $V^{\prime}$. 
L'existence d'une homotopie entre $\gamma_{0}$ et $\gamma_{1}$ fournit l'existence d'un anneau immergé $A=\phi\left(S^{1} \times[0,1]\right), \partial A=\gamma_{0} \cup \gamma_{1}$.

Par une homotopie de $A$, on se ramène à un anneau encore lisse, toujours noté $A$, mais qui est également transversal à $T^{2} \times\{a, b+2 k \pi\}$ et tel que toutes ses intersections avec $T^{2} \times\{a, b+2 k \pi\}$ soient non contractibles dans $V$. Pour obtenir cette deuxième propriété, on conjugue le fait que les tores $T^{2} \times\{a, b+$ $2 k \pi\}$ sont incompressibles avec le résultat d'un algorithme classique de Haken. A la source dans $S^{1} \times[0,1]$, les préimages par $\phi$ des courbes d'intersection forment une collection de courbes disjointes et parallèles. Elles découpent $S^{1} \times$ $[0,1]$ en une collection d'anneaux. On note $\delta_{1}, \ldots, \delta_{p-1}$ leur image par $\phi$ et $A_{1}, \ldots, A_{p}$ celle des anneaux. Par convention, $\gamma_{0}, \delta_{1} \subset A_{1}, \gamma_{1}, \delta_{p-1} \subset A_{p}$ et, pour $2 \leq k \leq p-1, \partial A_{k}=\delta_{k-1} \cup \delta_{k}$.

Par construction, $A_{1} \subset T^{2} \times[a, b+2 k \pi]$ et donc la pente de $\delta_{1}$ est identique à celle de $\gamma_{0}$. D'après le lemme 3.1] la pente de $\delta_{2}$ est soit la même que celle de $\delta_{1}$, soit $\delta_{1}$ et $\delta_{2}$ ont pour pente $\pm p_{a}$ et $\pm p_{b}$. Par hypothèse, ce n'est pas le cas pour $\gamma_{0}$, donc ce n'est également pas le cas pour $\delta_{1}$. On en déduit que la pente de $\delta_{2}$ est la même que celle de $\delta_{1}$. Une récurrence immédiate montre que, pour $1 \leq p-1$, le pente de $\delta_{k}$ est la même que celle de $\gamma_{0}$.

Si $\gamma_{1} \subset T^{2} \times\left([a, a+c] \cup\left[b-c^{\prime}+2 k \pi, b+2 k \pi\right]\right)$, alors on a aussi

$$
A_{p} \subset T^{2} \times\left([a, a+c] \cup\left[b-c^{\prime}+2 k \pi, b+2 k \pi\right]\right) .
$$

La pente de $\gamma_{1}$ est dans ce cas égale à celle de $\gamma_{0}$. Ceci met en contradiction le choix de $h$ et le fait que le champ de Reeb soit positivement transversal à un feuilletage $\mathcal{F}_{a} \times[a, a+c] \cup\left(-\mathcal{F}_{b}\right) \times\left[b-c^{\prime}+2 k \pi, b+2 k \pi\right]$.

Dans le cas où $\gamma_{1} \subset V^{\prime}$, on observe que $\left[\delta_{p-1}\right] \in H_{1}\left(V^{\prime}, \mathbb{Z}\right)$ a, dans $V^{\prime}$, une intersection négative avec $[S] \in H_{2}\left(V^{\prime}, \partial V^{\prime}, \mathbb{Z}\right)$. Comme $\left[\gamma_{1}\right]=\left[\delta_{p-1}\right]$, l'intersection de $\left[\gamma_{1}\right]$ avec $[S]$ est également négative. Ceci contredit le fait que toutes les intersections de $\gamma_{1}$ avec $S$ sont positives (car le champ de Reeb est positivement transversal à $S$ ).

Pour finir, si $\gamma_{1}$ est dans $T^{2} \times\left[a+c, b-c^{\prime}+2 k \pi\right]$, sa pente est la même que celle de $\gamma_{0}$.

Corollaire 3.3 Pour $\epsilon$ assez petit, si $V$ n'est pas un fibré en tores sur le cercle et si aucune composante de $V^{\prime} n^{\prime}$ 'est un fibré tordu en intervalles sur la bouteille de Klein, $H C_{0}^{h}\left(V, \xi_{\epsilon, k}\right) \simeq \mathbb{Z}^{k}$.

Démonstration On a exactement $k$ cercles d'orbites de $R_{\epsilon, k}$ dans la classe $h$ qui sont toutes situées dans $T^{2} \times\left[a+c, b-c^{\prime}+2 k \pi\right]$. Toutes les orbites de ces 
familles ont la même période. Par conséquent, les seuls cylindres holomorphes entre des orbites de la classe $h$ dans la symplectisation de $\left(V, \alpha_{\epsilon, k}\right)$ sont les cylindres verticaux. En s'inspirant de techniques à la Morse-Bott [2, on peut perturber la forme de contact $\alpha_{\epsilon, k}$ au moyen d'une fonction de Morse $f$ ayant exactement un maximum et un minimum sur chaque cercle d'orbites de la classe $h$, de sorte que chaque cercle donne lieu à deux orbites non dégénérées, l'une de degré -1 et l'autre de degré 0 . Ces orbites sont les générateurs du complexe de chaîne pour l'homologie de contact. Après la perturbation, les seuls cylindres holomorphes d'index 1 correspondent aux trajectoires du gradient de $f$. Leurs contributions à la différentielle du complexe de chaîne s'annulent donc deux à deux, et chaque générateur du complexe de chaîne donne lieu à un générateur de l'homologie de contact.

Dans le cas où une seule composante $V_{1}$ de $V^{\prime}$ (attachée par exemple le long de $\left.T^{2} \times\{b\}\right)$ est un fibré tordu en intervalles sur la bouteille de Klein, on se ramène au cas précédent par passage au revêtement. Soit en effet $\pi: T^{2} \times[-1,1] \rightarrow V_{1}$ une application de revêtement de degré 2 qui est compatible avec la forme de contact $\cos t d x-\sin t d y$ de $T^{2} \times[-1,1]$. Si $T^{2}=(\mathbb{R} / \mathbb{Z})^{2}$, la projection $\pi$ est le passage au quotient pour la relation d'équivalence: $(x, y, t) \sim(x,-y,-t)$.

On munit $V_{1}$ de la structure de contact quotient. On désigne par $V_{2}$ l'autre composante de $V^{\prime}$.

On note $\left(W, \eta_{\epsilon, k}\right)$ le revêtement de degré 2 de $\left(V, \xi_{\epsilon, k}\right)$ obtenu en collant deux copies du complémentaire de l'intérieur de $V_{1}$ dans $V$ au revêtement $\pi^{-1}\left(V_{1}\right)$. Dans $W$, le tore épais

$$
P=T^{2} \times[a, b+2 k \pi] \cup \pi^{-1}\left(V_{1}\right) \cup T^{2} \times[a, b+2 k \pi]
$$

joue le rôle de $T^{2} \times[a, b+2 k \pi]$ dans $V$ : la structure $\eta_{\epsilon, k}$ y est conjuguée à un produit $\left(T^{2} \times\left[a^{\prime}, b^{\prime}\right], \operatorname{ker}(\cos t d x-\sin t d y)\right), 4 k \pi \leq b^{\prime}-a^{\prime} \leq(4 k+2) \pi$. Le complémentaire de $P$ dans $W$ est constitué de deux copies de $V_{2}$ munies de la structure de contact $\operatorname{ker} \alpha_{\epsilon, 0}$.

D'après le théorème 2.1 pour tout $\epsilon>0$, il existe $c, c^{\prime}>0$ et une forme de contact $\beta_{\epsilon, k}$ pour $\eta_{\epsilon, k}$ qui vaut $\alpha_{\epsilon, 0}$ sur $W \backslash P, \cos t d x-\sin t d y$ sur $T^{2} \times$ $[a+c, b+2 k \pi] \cup \pi^{-1}\left(V_{1}\right) \cup T^{2} \times\left[a, b-c^{\prime}+2 k \pi\right]$ et dont le champ de Reeb est positivement transversal à un feuilletage $\mathcal{F}_{a} \times[a, a+c] \cup\left(-\mathcal{F}_{b}\right) \times\left[b-c^{\prime}, b\right]$ dont les deux pentes dans $P$ sont $\epsilon$-proches de celles des copies de $\partial S$ dans $\partial P$.

De plus, les pentes dans $P$ des deux copies du bord de $S$ situées de part et d'autre de $P$ sont opposées. 
Grâce à l'étude précédente, on montre que, pour $\epsilon$ assez petit, les structures $\eta_{\epsilon, k}$ sont deux à deux non isotopes dans $W$. C'est donc aussi le cas des structures de la famille $\left(\xi_{\epsilon, k}\right)_{k \in \mathbb{N}}$.

\subsection{La pente de $\partial S \cap T^{2} \times\{a\}$ est égale à celle de $\partial S \cap T^{2} \times\{b\}$}

Si $T$ sépare $V$, la surface $S$ se décompose elle aussi en deux composantes situées de part et d'autre de $T^{2} \times[a, b]$. Il suffit de changer l'orientation d'une des deux composantes de $S$ pour se ramener au cas précédent: on définit une nouvelle famille de structures $\left(\xi_{\epsilon, k}^{\prime}\right)_{k \in \mathbb{N}}$ deux à deux non isotopes.

Si $T$ ne sépare pas $V$, on modifie la structure $\xi_{\epsilon, k}$ sur $V$ en collant à la restriction de $\alpha_{\epsilon, 0}$ sur $\left.V \backslash T^{2} \times\right] a+c, b-c^{\prime}$ [ le produit $\left(T^{2} \times\left[a+c, b-c^{\prime}+(2 k+\right.\right.$ 1) $\pi], \cos t d x-\sin t d y)$. La structure $\xi_{\epsilon, k}^{\prime}$ ainsi obtenue n'est pas orientable. Elle le devient si on considère son rappel dans le revêtement $W$ de degré 2 de $V$ obtenu en collant deux copies de $\left.V \backslash T^{2} \times\right] a+c, b-c^{\prime}[$ à deux copies de $T^{2} \times\left[a+c, b-c^{\prime}+(2 k+1) \pi\right]$. Dans $W$, on récupère une forme de contact $\beta_{\epsilon, k}$ en prenant $\cos t d x-\sin t d y$ sur $\pi^{-1}\left(T^{2} \times\left[a+c, b-c^{\prime}+(2 k+1) \pi\right]\right)$ et, respectivement, $\alpha_{\epsilon, 0}$ et $-\alpha_{\epsilon, 0}$ sur les deux exemplaires de $\left.V \backslash T^{2} \times\right] a+c, b-c^{\prime}+(2 k+1) \pi[$.

On se fixe une composante $P$ de $\pi^{-1}\left(T^{2} \times\left[a+c, b-c^{\prime}+(2 k+1) \pi\right]\right)$ dans $W$. L'autre est notée $Q$. Le champ de Reeb associé à la forme $\beta_{\epsilon, k}$ est transversal aux deux rappels $S_{1}$ et $S_{2}$ de $S$, positivement dans un cas $\left(S_{1}\right)$ et négativement dans l'autre. On considère alors la surface $S^{\prime}=S_{1} \cup S_{2}$. On choisit $\epsilon$ petit pour que $H=S^{1} \backslash\left(C\left(\partial S^{\prime}\right) \cup C\left(\mathcal{F}_{a}\right) \cup C\left(-\mathcal{F}_{b}\right)\right)$ soit de cardinal infini. Comme précédemment, on note $p_{a}$ et $p_{b}$ les pentes des éventuelles composantes fibrées adjacentes à $P$ dans la décomposition de Jaco-ShalenJohanson du complémentaire de $P$ dans $W$. On fixe une classe homotopie libre $h$ d'une courbe de $P$ de pente dans $H \backslash\left\{ \pm p_{a}, \pm p_{b}\right\}$.

On montre, comme dans la partie précédente, que toute orbite du flot de Reeb de $\beta_{\epsilon, k}$ dans la classe $h$ doit provenir de l'une des préimages de $T^{2} \times[a+c, b-$ $\left.c^{\prime}+(2 k+1) \pi\right]$ (dans le cas qui nous intéresse, aucune composante de $V^{\prime}$ n'est un tore épaissi, un fibré tordu en intervalles sur une bouteille de Klein, ou un tore solide). De plus, les seules orbites de $Q$ homotopes à une orbite de $P$ sont homotopes à des orbites de pente $\pm p_{a}$ ou $\pm p_{b}$. En conséquence, on obtient le corollaire:

Corollaire 3.4 Pour $\epsilon>0$ assez petit, $H C_{0}^{h}\left(W, \operatorname{ker} \beta_{\epsilon, k}, \mathbb{Z}\right) \simeq \mathbb{Z}^{k}$.

On en déduit immédiatement que, pour $\epsilon$ fixé assez petit, les structures de la famille $\left(\xi_{\epsilon, k}^{\prime}\right)_{k \in \mathbb{N}}$ sont deux à deux non isotopes sur $V$. 


\subsection{Deux cas particuliers}

On traite ici le cas des fibrés en tores sur le cercle et des variétés obtenues par collage de deux fibrés tordus en intervalles sur la bouteille de Klein. Dans ces deux cas, les familles de structures sont explicites.

Comme une structure de contact sur un fibré tordu en intervalles sur la bouteille de Klein est le quotient par $\mathbb{Z}_{2}$ d'une structure de contact sur un fibré en tores sur le cercle, ces deux situations sont analogues. Nous ne traiterons donc que le cas où $V=(\mathbb{R} / \mathbb{Z})^{2} \times[0,1] / \sim$, avec les identifications $(x, y, 1) \sim(A(x, y), 0)$ pour $A \in S L(2, \mathbb{Z})$. Les structures de contact $\xi_{\epsilon, k}=\operatorname{ker} \alpha_{\epsilon, k}$ introduites dans la section 2 sont isotopes aux structures de contact $\xi_{k}=$ ker $\alpha_{k}$ définies par

$$
\alpha_{k}=\cos ((a+2 k \pi) z+b) d x+\sin ((a+2 k \pi) z+b) d y .
$$

Soit $A^{t}$ la transposée de la matrice $A$. Les nombres $0<a, b \leq 2 \pi$ sont choisis de sorte que $(\cos b, \sin b)=A^{t}(\cos (a+b), \sin (a+b))$.

On distingue trois cas selon la monodromie $A$ :

(i) Si $|\operatorname{tr} A|<2$, la monodromie $A$ est d'ordre fini dans $S L(2, \mathbb{Z})$. Par conséquent, toute classe d'homotopie libre $h$ dans $T^{2}$ ne contient qu'un nombre fini d'orbites de Reeb fermées.

(ii) Si $|\operatorname{tr} A|=2$, la monodromie $A$ est conjuguée à

$$
\pm\left(\begin{array}{cc}
1 & n \\
0 & 1
\end{array}\right)
$$

dans $S L(2, \mathbb{Z})$ pour un certain entier $n$. En particulier, il existe une classe d'homotopie libre $h$ dans $T^{2}$ ne contenant qu'un nombre fini et non nul d'orbites de Reeb fermées.

(iii) Si $|\operatorname{tr} A|>2$, la monodromie ne préserve aucune direction de pente rationnelle. Par conséquent, toute classe d'homotopie libre $h$ dans $T^{2}$ contient un nombre infini d'orbites de Reeb fermées, lorsque $k \geq 1$.

Pour calculer la différentielle du complexe pour l'homologie de contact, nous aurons besoin du lemme suivant.

Lemme 3.5 Soit $F:\left(\mathbb{R} \times S^{1}, j\right) \rightarrow(\mathbb{R} \times V, J)$ un cylindre holomorphe dans la symplectisation de $\left(V, \alpha_{k}\right)$ et aboutissant en $t=-\infty$ à une orbite de Reeb fermée dans $T^{2} \times\left\{z_{0}\right\}$. Alors le cylindre $F\left(\mathbb{R} \times S^{1}\right)$ est contenu dans $\mathbb{R} \times T^{2} \times$ $\left\{z_{0}\right\}$. 
Démonstration Supposons par contradiction qu'il existe $p \in \mathbb{R} \times S^{1}$ tel que $F(p) \notin \mathbb{R} \times T^{2} \times\left\{z_{0}\right\}$. Sans perte de généralité, on peut supposer que $z \circ$ $F(p)=z_{0}-\epsilon$ ou $z_{0}+\epsilon$, pour un petit $\epsilon>0$. Pour fixer les idées, on choisit $z \circ F(p)=z_{0}+\epsilon$.

Par le théorème de Sard, on peut choisir deux valeurs régulières $z_{1}$ et $z_{2}$ de la fonction $z \circ F$ sur $\mathbb{R} \times S^{1}$, satisfaisant $z_{0}<z_{1}<z_{2}<z_{0}+\epsilon$. Comme il n'y a pas d'orbite de Reeb fermée de classe $h$ dans $T^{2} \times\left[z_{1}, z_{2}\right]$ pour $\epsilon>0$ suffisamment petit, le sous-ensemble $C_{z_{1}, z_{2}}=F^{-1}\left(\mathbb{R} \times T^{2} \times\left[z_{1}, z_{2}\right]\right)$ du cylindre $\mathbb{R} \times S^{1}$ est non vide, compact et bordé de lacets simples et lisses. De plus, les images de ces lacets dans $\mathbb{R} \times V$ sont soit contractibles, soit de classe $h$.

Par le théorème de Stokes, on a

$$
\int_{C_{z_{1}, z_{2}}} F^{*} d \alpha_{k}=\int_{F^{-1}\left(\mathbb{R} \times T^{2} \times\left\{z_{2}\right\}\right)} F^{*} \alpha_{k}-\int_{F^{-1}\left(\mathbb{R} \times T^{2} \times\left\{z_{1}\right\}\right)} F^{*} \alpha_{k} .
$$

Ces deux intégrales sont aisément calculables, puisque la 1 -forme $\alpha_{k}$ est fermée sur les sous-variétés $T^{2} \times\left\{z_{1}\right\}$ et $T^{2} \times\left\{z_{2}\right\}$. Leur différence est nulle ou du signe de $\cos \left((a+2 k \pi)\left(z_{2}-z_{0}\right)\right)-\cos \left((a+2 k \pi)\left(z_{1}-z_{0}\right)\right)<0$ selon que la classe d'homologie de $F^{-1}\left(\mathbb{R} \times T^{2} \times\left\{z_{1}\right\}\right)$ est nulle ou correspond à $h$.

D'autre part, comme $F$ est holomorphe, on doit avoir $F^{*} d \alpha_{k} \geq 0$ sur $\mathbb{R} \times$ $S^{1}$. Par conséquent, la 2 -forme $d \alpha_{k}$ doit s'annuler sur $C_{z_{1}, z_{2}}$, de sorte que $F$ est un cylindre vertical au-dessus d'une orbite de Reeb. On obtient ainsi une contradiction.

Comme dans le corollaire 3.3. on déduit de ce lemme que la différentielle du complexe définissant l'homologie de contact $H C_{0}^{h}\left(V, \xi_{k}\right)$ est identiquement nulle. Par conséquent, si $h$ est une classe d'homotopie libre dans $V$ contenant un nombre fini et non nul d'orbites de Reeb fermées, alors le rang de $H C_{0}^{h}\left(V, \xi_{k}\right)$ dépend de $k$. Plus précisément, si $m$ désigne le plus petit entier strictement positif tel que $A^{m} h=h$, alors

$$
\operatorname{rang} H C_{0}^{h}\left(V, \xi_{k+1}\right)=\operatorname{rang} H C_{0}^{h}\left(V, \xi_{k}\right)+m .
$$

En particulier, dans les cas (i) et (ii), les structures de contact $\xi_{k}$ sont non isotopes pour des valeurs différentes de $k$.

Si $h$ est une classe d'homotopie libre dans $V$ contenant un nombre infini d'orbites de Reeb fermées, l'homologie de contact est de rang infini pour tout $k$ et ne permet pas de conclure. On utilise alors l'homologie de contact en conjonction avec la fonctionnelle d'action, qui associe à toute orbite de Reeb fermée sa période. Soit $\alpha$ une forme de contact pour une structure de contact $\xi$ sur $V$; 
on note $H C_{*}^{h, T}(V, \alpha)$ l'ensemble des classes d'homologie dans $H C_{*}^{h}(V, \alpha)$ ayant un représentant de période inférieure ou égale à $T$. Notons que ceci dépend du choix de la forme de contact $\alpha$ pour $\xi$. On définit

$$
I_{*}^{h}(V, \alpha)=\lim _{T \rightarrow \infty} \frac{\operatorname{rang} H C_{*}^{h, T}(V, \alpha)}{\ln T}
$$

lorsque la limite existe. Dans le cas contraire, il est encore possible de définir $\underline{I}_{*}^{h}(V, \alpha)$ et $\bar{I}_{*}^{h}(V, \alpha)$ en utilisant une limite inférieure ou supérieure respectivement.

Lemme 3.6 La quantité $I_{*}^{h}(V, \alpha)$ ne dépend que de la classe d'isotopie de $\xi$.

Démonstration Considérons la forme de contact $c \alpha$ pour $c>0$. On a

$$
\begin{aligned}
I_{*}^{h}(V, c \alpha) & =\lim _{T \rightarrow \infty} \frac{\operatorname{rang} H C_{*}^{h, T}(V, c \alpha)}{\ln T} \\
& =\lim _{T \rightarrow \infty} \frac{\operatorname{rang} H C_{*}^{h, \frac{T}{c}}(V, \alpha)}{\ln T} \\
& =\lim _{T \rightarrow \infty} \frac{\operatorname{rang} H C_{*}^{h, \frac{T}{c}}(V, \alpha)}{\ln \frac{T}{c}} \frac{\ln T-\ln c}{\ln T} \\
& =I_{*}^{h}(V, \alpha) .
\end{aligned}
$$

Soient $\alpha_{1}$ et $\alpha_{2}$ deux formes de contact sur $V$. On dira que $\alpha_{1}<\alpha_{2}$ si on peut munir $\mathbb{R} \times V$ d'une forme symplectique $\omega$ exacte telle que $\omega=d\left(e^{t} \alpha_{2}\right)$ lorsque $t \leq 0$ et $\omega=d\left(e^{t-t_{0}} \alpha_{1}\right)$ lorsque $t \geq t_{0}$, pour un certain $t_{0}>0$. Si $\alpha_{1}$ et $\alpha_{2}$ sont des formes de contact pour des structures de contact isotopes sur $V$ alors il existe $c>0$ tel que $c \alpha_{1}<\alpha_{2}$.

Si $\alpha_{1}<\alpha_{2}$ alors rang $H C_{*}^{h, T}\left(V, \alpha_{2}\right) \leq \operatorname{rang} H C_{*}^{h, T}\left(V, \alpha_{1}\right)$. En effet, en vertu des propriétés fonctorielles de l'homologie de contact [1], au cobordisme symplectique $(\mathbb{R} \times V, \omega)$ correspond un isomorphisme

$$
\Phi: H C_{*}^{h}\left(V, \alpha_{2}\right) \rightarrow H C_{*}^{h}\left(V, \alpha_{1}\right)
$$

tel que $\Phi\left(H C_{*}^{h, T}\left(V, \alpha_{2}\right)\right) \subset H C_{*}^{h, T}\left(V, \alpha_{1}\right)$. Par conséquent, si $\alpha_{1}<\alpha_{2}$ alors $I_{*}^{h}\left(V, \alpha_{2}\right) \leq I_{*}^{h}\left(V, \alpha_{1}\right)$.

La conclusion suit de ces deux propriétés de $I_{*}^{h}$.

En vertu de ce résultat, nous utiliserons plutôt la notation $I_{*}^{h}(V, \xi)$, qui ne fait plus apparaître la forme de contact $\alpha$. 
Calculons maintenant $I_{0}^{h}\left(V, \xi_{k}\right)$ lorsque $|\operatorname{tr} A|>2$, en considérant plutôt la variété de contact $\left(T^{2} \times[0,1], \xi_{k}\right)$. La classe d'homotopie libre $h$ dans $V$ correspond aux classes d'homotopie libres $A^{n} h$ dans $T^{2} \times[0,1]$, pour $n \in \mathbb{Z}$. Il y a $k$ ou $k+1$ orbites de Reeb fermées dans la classe d'homotopie libre $A^{n} h$; cette alternative ne dépend que de $\xi_{0}$ lorsque $|n|$ est suffisamment grand. L'action de ces orbites est donnée par $\left\|A^{n} h\right\| \simeq C \lambda^{|n|}$ lorsque $|n|$ est grand, où $\lambda>1$ est l'une des valeurs propres de $A$. On en déduit que $I_{0}^{h}\left(V, \xi_{k}\right)$ est égal à $\frac{2 k}{\ln \lambda}$ ou $\frac{2(k+1)}{\ln \lambda}$. En particulier, les structures de contact $\xi_{k}$ ne sont pas isotopes pour différentes valeurs de $k \geq 0$.

\section{Généralisations}

L'étude proposée ci-dessus s'adapte dans le cas où la torsion est non nulle dans plusieurs classes d'isotopies.

Plus précisemment, soient $T_{0}, \ldots, T_{n}$ des tores incompressibles deux à deux disjoints et non parallèles dans une variété close et irréductible $V$. On note $\left.T_{i} \times\right] a_{i}, b_{i}$ [ un voisinage tubulaire de $T_{i}$ dans $V$ (choisis deux à deux disjoints) et $S$ une surface minimale essentielle coorientée dans $V^{\prime}=V \backslash \cup_{i}\left(T_{i} \times\right] a_{i}, b_{i}[)$ qui rencontre toutes les composantes de $\partial V^{\prime}$.

Comme dans le théorème 2.1] on munit $V$, pour $\epsilon>0$, d'une forme de contact $\alpha_{\epsilon}$, dont le champ de Reeb $R_{\epsilon}$ possède, pour tout $0 \leq i \leq n$, les propriétés suivantes:

- $R_{\epsilon}$ est positivement transversal à $S$, tangent à $T_{i} \times\left\{a_{i}, b_{i}\right\}$;

- $R_{\epsilon}$ vaut $\cos t \partial_{x}-\sin t \partial_{y} \operatorname{sur} T_{i} \times\left[a_{i}+c, b_{i}-c^{\prime}\right]\left(\alpha_{\epsilon}\right.$ vaut $\left.\cos t d x-\sin t d y\right)$;

- $\operatorname{sur} T_{i} \times\left[a_{i}, a_{i}+c\right]$ et $T_{i} \times\left[b_{i}-c^{\prime}, b_{i}\right], R_{\epsilon}$ est positivement transversal à un feuilletage en anneau $\mathcal{F}_{a_{i}} \times\left[a_{i}, a_{i}+c\right] \cup\left(-\mathcal{F}_{b_{i}}\right) \times\left[b_{i}-c^{\prime}, b_{i}\right]$, où $\mathcal{F}_{a_{i}}$ et $\mathcal{F}_{b_{i}}$ sont des feuilletages en cercles de $T_{i} \times\left\{a_{i}\right\}$ et $T_{i} \times\left\{b_{i}\right\}$ dont les pentes sont $\epsilon$-proches de celles d'une composante de, respectivement, $\partial S \cap T_{i} \times\left\{a_{i}\right\}$ et $\partial S \cap T_{i} \times\left\{b_{i}\right\}$.

Sur chaque produit $\left.T_{i} \times\right] a_{i}, b_{i}\left[\right.$, on remplace le produit de contact $\left(T_{i} \times\left[a_{i}+\right.\right.$ $\left.\left.c, b_{i}-c^{\prime}\right], \cos t d x-\sin t d y\right)$ par $\left(T_{i} \times\left[a_{i}+c, b_{i}-c^{\prime}+2 k_{i} \pi\right], \cos t d x-\sin t d y\right)$, pour un certain $k_{i} \in \mathbb{N}$. On obtient ainsi une certaine forme de contact $\alpha_{\epsilon, k_{0}, \ldots, k_{n}}$.

En reprenant le même raisonnement que précédemment, et avec les notations introduites ci-dessus, on montre le théorème suivant: 
Théorème 4.1 Si, pour un certain $0 \leq i \leq n, S \cap T_{i} \times\left\{a_{i}\right\}$ et $S \cap T_{i} \times\left\{b_{i}\right\}$ ne sont pas de pente opposée (en projection dans $T_{i}$ ) et si $k_{i} \geq 1$, alors il existe une classe d'homotopie $h_{i}$ de courbe dans $T_{i}$ pour laquelle:

$$
H C_{*}^{h_{i}}\left(V, \xi_{k_{0}, \ldots, k_{n}}, \mathbb{Z}\right) \simeq \mathbb{Z}^{k_{i}} .
$$

En particulier, la structure $\xi_{k_{0}, \ldots, k_{n}}$ vérifie la conjecture de Weinstein.

Tous les exemples de structures de contact universellement tendues connus sont de ce type. La restriction principale dans l'énoncé du théorème 4.1 est topologique et porte sur la pente de $\partial S \cap T_{i} \times\left\{a_{i}, b_{i}\right\}$.

\section{Références}

[1] C Abbas, K Cieliebak, H Hofer, The Weinstein conjecture for planar contact structures in dimension three, préprint, arXiv:math.SG/0409355

[2] F Bourgeois, A Morse-Bott approach to contact homology, from: "Symplectic and contact topology: interactions and perspectives", Fields Inst. Commun. 35, Amer. Math. Soc. (2003) 55-77 MathReview

[3] F Bourgeois, A Morse-Bott approach to contact homology, thèse de doctorat, Stanford University, 2002

[4] F Bourgeois, Y Eliashberg, H Hofer, K Wysocki, E Zehnder, Compactness results in symplectic field theory, Geom. Topol. 7 (2003) 799-888 MathReview

[5] F Bourgeois, K Mohnke, Coherent orientations in symplectic field theory, Math. Z. 248 (2004) 123-146 MathReview

[6] V Colin, Structures de contact tendues sur les variétés torö̈dales, Ann. Scient. Ec. Norm. Sup. 34 (2001) 267-286 MathReview

[7] V Colin, Une infinité de structures de contact tendues sur les variétés toroïdales, Comment. Math. Helv. 76 (2001) 353-372 MathReview

[8] V Colin, E Giroux, K Honda, Finitude homotopique et isotopique des structures de contact tendues, en préparation

[9] V Colin, K Honda, Constructions controlées de champs de Reeb, en préparation

[10] D Dragnev, Fredholm theory and transversality for noncompact pseudoholomorphic maps in symplectizations, Comm. Pure Appl. Math. 57 (2004) 726-763 MathReview

[11] Y Eliashberg, A Givental, H Hofer, Introduction to symplectic field theory, Geom. Funct. Anal. Special Volume (2000) Part II, 560-673 MathReview 
[12] E Giroux, Une infinité de structures de contact sur une infinité de variétés, Inv. Math. 135 (1999) 789-802 MathReview

[13] H Hofer, Pseudoholomorphic curves in symplectizations with application to the Weinstein conjecture in dimension three, Invent. Math. 114 (1993) 515-563 MathReview

[14] K Honda, W Kazez, G Matic̀, Convex decomposition theory, Int. Math. Res. Not. 2 (2002) 55-88 MathReview

[15] H Jaco, P Shalen, Seifert fibered spaces in 3-manifolds, Memoirs of the AMS, 21 (1979) no. 220 MathReview 